\title{
REFERENCES
}

1. H. Cartan, Fonctions theta sur le tore I, II, mimeographed seminar notes on Several Complex Variables, Paris, 1951-1952.

2. S. Chern, Complex manifolds, mimeographed lecture notes, University of Chicago, 1955-1956.

3. A. Comessatti, Sugl'indici di singolarita a piu dimensioni della varieta abeliane, Rendiconti del Seminario Matematico della Università di Padova, vol. 5 (1934) p. 50.

4. B. Eckmann and H. Guggenheimer, Formes differentielles et metrique hermitienne sans torsion, Comptes Rendus vol. 229 (1949) pp. 464-466, 489-491.

5. W. V. D. Hodge, Harmonic integrals, Cambridge, 1952, $\S 43,51$.

Massachusetts Institute of Technology

\section{CUT SETS IN TOTALLY NONAPOSYNDETIC CONTINUA ${ }^{1}$}

\author{
EDWARD E. GRACE
}

For metric spaces, F. Burton Jones has shown [1, Theorem 12] that if $D$ is an open subset of a compact continuum $M$ such that $M$ is nonaposyndetic at every point of $D$, then $D$ contains a point $x$ and $M$ contains points $y$ and $z$ such that $y$ cuts $x$ from $z$ in $M$. This paper extends the results of Jones to a certain class of topological spaces, gives a cut set in some cases where there is no cut points and gives stronger cutting properties.

Definition. ${ }^{2}$ If $A$ and $B$ are two mutually exclusive subsets of a topological continuum (i.e., a connected topological space) $T$ and there does not exist a continuum $T^{\prime}$ and an open subset $U$ of $T$ such that $[T-A] \supset T^{\prime} \supset U \supset B$, then $T$ is nonaposyndetic at $B$ with respect to $A$.

Definition. If $T$ is a topological continuum, $A$ is a subset of $T$ and $G$ is a collection of subsets of $T$ and if, for each point $x$ of $A$, there is a member $g$ of $G$ such that $T$ is nonaposyndetic at $x$ with respect to $g$, then $T$ is totally nonaposyndetic on $A$ with respect to $G$. If $A=T$ then $T$ is totally nonaposyndetic with respect to $G$. If, in addition, $G=\{\{x\} \mid x$ is in $T\}$ then $T$ is totally nonaposyndetic.

Definition. If $T$ is a topological continuum, $Z$ is the smallest cardinal number of a topological basis for $T$ and the subset $A$ of $T$

Presented to the Society, August 29, 1957; received by the editors June 25, 1957.

1 This paper is a generalization of part of a dissertation submitted to the Graduate School of the University of North Carolina in partial fulfillment of the requirements for the Ph.D. degree. The author wishes to express his appreciation to Professor F. Burton Jones for valuable suggestions.

2 The first two definitions are generalizations of definitions due to Jones [1]. Terms not defined herein are used as in [1] or [3]. 
is the nonvacuous common part of $Z$ open subsets of $T$, then $A$ is a domain intersection subset of $T$. If $A$ is the nonvacuous common part of $Z$ dense, open subsets of $T$, then $A$ is a dense-domain intersection subset of $T$

The reader should note that a domain intersection subset of a perfectly separable topological continuum is an inner limiting set ( $G_{\delta}$ set) relative to that continuum.

Definition. If (1) $Z$ is a cardinal number, (2) $G$ is a collection of proper subsets of a topological continuum $T$, and (3) $E$ is a collection of open sets the cardinality of which is $Z$ if $Z$ is transfinite and finite if $Z$ is finite, such that, if $g$ is a member of $G$ and $T_{1}$ is a continuum in $T-g$, then there is a member $E^{\prime}$ of $E$ such that $T-T_{1} \supset E^{\prime} \supset g$; then $G$ is said to have the $Z$-domain property (relative to $T$ ) and $E$ is said to be a $Z$-domain collection for $G$ (relative to $T$ ).

It should be noted that if $G$ is a collection of nondense subsets of a topological continuum $T$ which has a basis of cardinality $Z$, then $G$ has the $Z$-domain property if either $Z$ is finite or each member of $G$ is a bicompact set.

Definition. ${ }^{3}$ If $T$ is a topological continuum and there is a topological basis for $T$ of cardinality $Z^{\prime}$ such that $T$ is not the sum of $Z^{\prime}$, or less than $Z^{\prime}$, closed subsets of $T$, each nowhere dense in $T$, then $T$ is a Baire topological continuum. If, in addition, $D$ is an open subset of $T$ and $D$ is not contained in the sum of $Z^{\prime}$, or less than $Z^{\prime}$, closed subsets of $T$, each nowhere dense in $T$, then $T$ is Baire topological on $D$. If, furthermore, $x$ is a point of $T$ and for each open subset $U$ of $T$ containing $x$, the continuum $T$ is Baire topological on $U$, then $T$ is Baire topological at $x$. If $T$ is Baire topological at each point of a subset $A$ of $T$, then $T$ is said to be locally Baire topological on $A$.

A complete, perfectly separable, metric continuum is a locally Baire topological continuum.

Standing notation. The point set $T$ is a Baire topological continuum. If $A$ is a subset of $T$ then $K(A)$ is the set of all points $x$ of $T$ such that $x$ is in $A$ or $T$ is nonaposyndetic at $x$ with respect to $A$. The smallest cardinal number of a basis for $T$ is $Z$. The first ordinal number that is preceded by $Z$ ordinals is $z$. The collection $G$ of proper subsets of $T$ has the $Z$-domain property and $E$ is a $Z$-domain collection for $G$. The cardinality of $E$ is $W$. The point set $P$ is a subset of $T$ of cardinality $V$ such that if $g$ is in $G$ then $P \cdot[T-g] \neq 0$. The sequence $p(1)$, $p(2), \cdots, p(i), \cdots$, is a most economical well-ordering of $P$. If $A$

${ }^{3}$ Compare with the properties, for a continuum, of being of the second category in itself and of being of the second category in itself at each point. 
is a subset of $T$ which does not contain $P$, then $F(A)$ is the first term of $P$ in $T-A$.

Lemma. No open subset of a topological continuum is contained in the sum of a finite number of closed sets, each nowhere dense in the continuum.

Theorem 1. If $T$ is Baire topological on the open set $D$ and $I$ is a dense-domain intersection subset of $T$, then the closure $\bar{I}$ of I contains an open subset of $D$.

Proof. Assume $\bar{I}$ does not contain an open subset of $D$. Then $\bar{D} \cdot \bar{I}$ is a nowhere dense closed set. The complement of $I$ is contained in the sum of $Z$ or less than $Z$ closed, nowhere dense sets and $\bar{D} \cdot \bar{I}$ $\supset D \cdot I$. Therefore $D$ is contained in the sum of $Z+1$ or less than $Z+1$ closed, nowhere dense sets. By the lemma, $Z+1$ cannot be finite. Hence $Z+1=Z$, and $D$ is contained in the sum of $Z$ or less than $Z$ closed, nowhere dense sets. This contradicts the choice of $D$. Therefore $\bar{I}$ contains an open subset of $D$

THEOREM 2. T contains a dense-domain intersection subset I such that if $x$ is a point of $I$ and $T$ is nonaposyndetic at $x$ with respect to the set $g$ of $G$, then $g$ cuts $x$ from $F(g)$ in $T$.

Proof. Either $W=Z$ or $W$ is finite. In either case $T$ is not the sum of $W$ closed, nowhere dense subsets of $T$. Let $w$ be the first ordinal that is preceded by $W$ ordinals. Let $E_{1}, E_{2}, \cdots, E_{i}, \cdots$, for $i<w$, be a most economical well-ordering of $E$. For $i<w$, let $H_{i}$ be the set of all points $x$ in $T$ such that either (1) $x$ is in $E_{i}$ or (2) $x$ is not in $K\left(E_{i}\right)$ or (3) $x$ is not in the $F\left(E_{i}\right)$-component of $T-E_{i}$.

The set $H_{i}$ is a dense open subset of $T$. It is open since it is defined as the sum of open sets. If it were not dense in $T$, there would be a point $y$ in $\left[T-E_{i}\right] \cdot K\left(E_{i}\right)$ and an open subset $u$ of $T$ such that $\left[T-E_{i}\right] \supset\left[\right.$ the $F\left(E_{i}\right)$-component of $\left.T-E_{i}\right] \supset u \supset y$. But this contradicts the definition of $K\left(E_{i}\right)$.

Let $I=\prod_{i<w} H_{i}$. The set $I$ is a dense-domain intersection subset of $T$. Assume there is a point $x$ of $I$ and a member $g$ of $G$ such that $T$ is nonaposyndetic at $x$ with respect to $g$ and $g$ does not cut $x$ from $F(g)$ in $T$. Then there is a continuum $H$ such that $[T-g] \supset H$ $\supset[x+F(g)]$. There is an open set $E_{j}$ in $E$ such that $[T-H] \supset E_{j} \supset g$. But $T$ is nonaposyndetic at $x$ with respect to $g$ and, consequently, $T$ is nonaposyndetic at $x$ with respect to $E_{j}$. Also, $E_{j} \cdot F(g)=0$ and so $F(g)=F\left(E_{j}\right)$. Therefore, $x$ is in the $F\left(E_{j}\right)$-component of $T-E_{j}$. But this means $x$ is not in $H_{j}$ and consequently not in $I$. This is a contradiction. Therefore if $x$ is in $I$ and $T$ is nonaposyndetic at $x$ 
with respect to the set $g$ in $G$, then $g$ cuts $x$ from $F(g)$ in $T$.

THEOREM 3. The intersection of $Z$ or less than $Z$ dense-domain intersection subsets of $T$ is a dense-domain intersection set.

If $Z$ is finite, Theorem 3 follows from the lemma. If $Z$ is transfinite, Theorem 3 follows from the fact that the sum of $Z$ or less than $Z$ collections, each containing $Z$ or less than $Z$ elements, contains not more than $Z$ elements.

THEOREM 4. If $D$ is an open set and $J$ is a dense-domain intersection subset of $T$ such that $T$ is Baire topological on $D$ and $T$ is totally nonaposyndetic on $D \cdot J$ with respect to $G$, then there is a dense-domain intersection set I contained in $J$ such that $\bar{I}$ contains some open subset of $D$ and if $x$ is in $I$ and $T$ is nonaposyndetic at $x$ with respect to $g$ of $G$, then $g$ cuts $x$ from $F(g)$ in $T$.

Proof. By Theorem 2, there is a dense domain intersection set $I^{\prime}$ such that if $x$ is in $I^{\prime}$ and $T$ is nonaposyndetic at $x$ with respect to $g$ of $G$, then $g$ cuts $x$ from $F(g)$ in $T$. By Theorem 3 (if $Z=1$, then $I^{\prime}=J$ ), the intersection $I$ of $J$ and $I^{\prime}$ is a dense-domain intersection subset of $T$. From Theorem 1 it follows that $\bar{I}$ contains some open subset of $D$.

Theorem 5. If the cardinality of $P$ is not greater than $Z$, then $T$ contains a dense-domain intersection set $I$ such that if $x$ is a point of $I$ and $T$ is nonaposyndetic at $x$ with respect to $g$ of $G$, then $g$ cuts $x$ from each point of $(P+g)-g$ in $T$.

Proof. The ordinal number $v$ is the first ordinal number that is preceded by $V$ ordinals. If $i<v$ and $j<v, i \neq 1$ and $i \neq j$, then let $p_{j}(i)=p(i), p_{j}(j)=p(1)$ and $p_{j}(1)=p(j)$. If $A$ is a subset of $T$ that does not contain $P$, then let $F_{j}(A)$ be the first term of the series $p_{j}(1), p_{j}(2), \cdots, p_{j}(k), \cdots$, for $k<v$, which is in $T-A$.

By Theorem 2, there is a dense-domain intersection subset $I_{j}$ of $T$ such that if $x$ is in $I_{j}$ and $T$ is nonaposyndetic at $x$ with respect to $g$ of $G$ then $g$ cuts $x$ from $F_{j}(g)$ in $T$. By Theorem 3, the set $I=\prod_{j<v} I_{j}$ is a dense-domain intersection subset of $T$.

If $x$ is in $I$ and $T$ is nonaposyndetic at $x$ with respect to $g$ of $G$, then $g$ cuts $x$ from each point of $(P+g)-g$. Otherwise, if $j$ were an ordinal such that $p(j) \cdot g=0$ and $g$ did not cut $x$ from $p(j)$, then, since $p(j)=p_{j}(1)=F_{j}(g)$, the set $g$ would not cut $x$ from $F_{j}(g)$ and, consequently, $x$ would not belong to $I_{j}$.

Theorem 6. If the cardinality of $P$ is not greater than $Z$, the set $D$ is open, and $J$ is a dense-domain intersection subset of $T$ such that $T$ is 
Baire topological on $D$ and $T$ is totally nonaposyndetic on $D \cdot J$ with respect to $G$; then there is a dense-domain intersection set $I$ contained in $J$ such that $\bar{I}$ contains some open subset of $D$ and if $x$ is in $I$ and $T$ is nonaposyndetic at $x$ with respect to $g$ of $G$, then $g$ cuts $x$ from each point of $(P+g)-g$ in $T$.

Theorem 6 follows from Theorem 4 by a construction similar to that in the proof of Theorem 5.

Theorem 7 (Theorem 8). Under the hypothesis of Theorem 4 (Theorem 6), if $T$ is locally Baire topological on $D$, then the closure of $I$, in the conclusion of Theorem 4 (Theorem 6), contains $D$.

Corollary 1. If (1) $M$ is a perfectly separable, complete, metric continuum, (2) $D$ is an open set such that $M$ is nonaposyndetic at each point of $D$, and (3) $Q$ is a countable subset of $M$, then $D$ contains an inner limiting subset $I$ of $M$, dense in $D$, such that if $x$ is in $I$ and $M$ is nonaposyndetic at $x$ with respect to the point $y$, then $y$ cuts $x$ from each point of $(Q+y)-y$ in $M$.

Corollary 2. If $M$ is a totally nonaposyndetic, compact, metric continuum, then $M$ contains a weak cut point.

Corollary 3. If $M$ is a compact, metric continuum which is totally nonaposyndetic with respect to some collection $H$ of closed sets, then some member of $H$ cuts $M$ (weakly).

This theory gives information about cut points in finite, connected spaces.

EXAMPLE 1. Let $T$ be the connected topological space consisting of the three points 0,1 , and 2 and the three regions $\{2\},\{1,2\}$, and $\{0,2\}$. Let $G$ consist of the sets $\{0\},\{1\}$, and $\{2\}$. Let $E$ be the collection of regions. $E$ is a minimal basis for $T$. Let $P=T$. Each member of $G$ consists of one point, so $G$ has the 3-domain property, $E$ is a 3 -domain collection for $G$, and $P$ has a point in the complement of each of the members of $G$. The continuum $T$ is nonaposyndetic at each point with respect to each member of $G$ not containing the point. Since $T$ has a finite basis, $T$ is a locally Baire topological continuum. Hence, the hypothesis of Theorem 8 is satisfied and therefore $T$ contains a dense-domain intersection subset $I$, dense in $T$, such that if $x$ is in $I$ and $T$ is nonaposyndetic at $x$ with respect to $g$ of $G$ then $g$ cuts $x$ from each point of $T-g$. But 2 is not in the closure of $T-2$ so 2 is in $I$. In addition, $T$ is nonaposyndetic at 2 with respect to $\{0\}$ and $\{1\}$ of $G$. Therefore 0 cuts 2 from 2 and from 1 and 1 cuts 2 from 2 and from 0. 
That this theory sometimes gives a cut set when there is no cut point is illustrated by the continuum $T$ described in Example 2 and indicated by Figure 1.

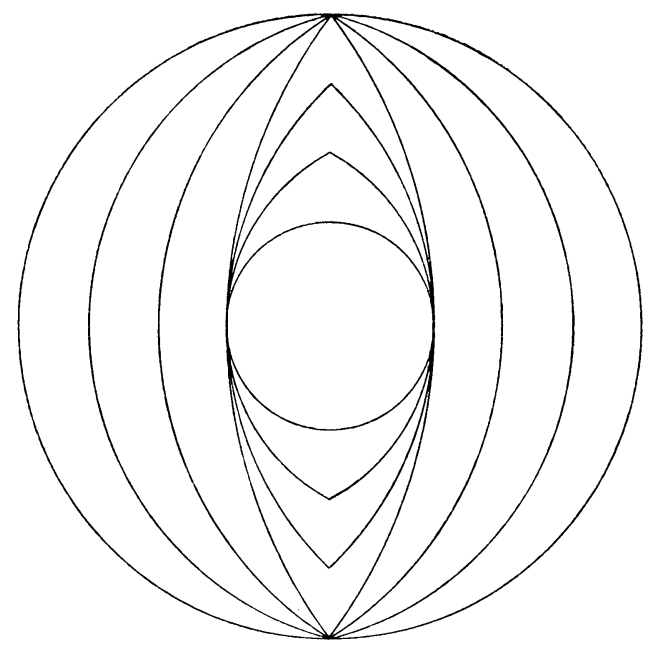

FIG. 1

Example 2. Let $C$ be the set of numbers in the interval from 0 to 1 that can be written to the base 3 using only the digits 0 and 2. Let $C^{\prime}$ be the set of all numbers of the form $x-1 / 2$, for $x$ in $C$. $T$ is the sum of the following arcs of circles: (1) for each $x$ in $C^{\prime}$, the arcs from $(0, x)$ to $(0,-x)$ through $(1 / 6,0)$ and through $(-1 / 6,0)$; and $(2)$ for each $x$ in $C^{\prime}$, the arc from $(0,1 / 2)$ to $(0,-1 / 2)$ through $(x, 0)$.

$T$ is aposyndetic, in fact each closed half plane with $(0,0)$ in it intersects $T$ in a continuum, and $T$ does not contain a weak cut point. However, $T$ is totally nonaposyndetic with respect to the collection of pairs of points of $T$; in fact, if $x$ is a point of $T$, then either $T$ is nonaposyndetic at $x$ with respect to $\{(0,1 / 2),(0,-1 / 2)\}$ or $T$ is nonaposyndetic at $x$ with respect to $\{(1 / 6,0),(-1 / 6,0)\}$. But $T$ is a compact metric continuum. Therefore, by Corollary 3 , some pair of points of $T$ cuts $T$.

The theorems of this paper are strongly dependent on the Baire topological condition.

EXAmple 3. There is a connected topological space $T^{\prime}$ which is totally nonaposyndetic but which does not contain a weak cut point.

Let $a_{0}$ and $a_{1}$ be two points of three dimensional Euclidean space. Let $A_{11}, A_{12}$, and $A_{13}$ be three mutually exclusive arc segments from 
$a_{0}$ to $a_{1}$. Let $A_{1}=a_{0}+a_{1}+\sum_{i=1}^{3} A_{1 i}$. Let $a_{11}, a_{12}$ and $a_{13}$ be points of $A_{11}, A_{12}$ and $A_{13}$ respectively. If $n$ is a positive integer, let $a_{n+1}$ be a point not in $\sum_{i=1}^{n} A_{i}$. For $i=1,2,3$, let $A_{(n+1) i}$ be an arc segment from $a_{n i}$ to $a_{n+1}$ in the complement of $\sum_{i=1}^{n} A_{i}$ and let $a_{(n+1) i}$ be a point in $A_{(n+1) i}$ such that $A_{(n+1) 1}, A_{(n+1) 2}$, and $A_{(n+1) 3}$ are mutually exclusive and each point of $\sum_{i=1}^{n} A_{i}$ is within the distance $1 / n$ of some point of $A_{(n+1) i}$ between $a_{n i}$ and $a_{(n+1) i}$. Let $A_{n+1}=a_{n+1}+\sum_{i=1}^{3}\left[a_{n i}+A_{(n+1) i}\right]$ and let $T^{\prime}=\sum A_{i}$. The space $T^{\prime}$ is a connected subspace of a perfectly separable, metric space so $T^{\prime}$ is a connected topological space. Not only is $T^{\prime}$ totally nonaposyndetic, but $T^{\prime}$ is nonaposyndetic at each point of $T^{\prime}$ with respect to each other point of $T^{\prime \prime}$. Each point of a Baire topological continuum having these properties is a weak cut point of the continuum. However, the perfectly separable, metric space $T^{\prime}$ contains no weak cut point. In fact, if $x$ is a point of $T^{\prime}$ then $T^{\prime}-x$ is arcwise connected.

\section{BIBLIOGRAPHY}

1. F. B. Jones, Concerning non-aposyndetic continua, Amer. J. Math. vol. 70 (1948) pp. 403-413.

2. - Concerning aposyndetic and non-aposyndetic continua, Bull. Amer. Math. Soc. vol. 58 (1952) pp. 137-151.

3. R. L. Moore, Foundations of point set theory, Amer. Math. Soc. Colloquium Publications, vol. 13, 1932.

4. G. T. Whyburn, Analytic topology, Amer. Math. Soc. Colloquium Publications. vol. $28,1942$.

The University of North Carolina and

EMORY UNIVERSITY 